\title{
Children's use of semantic organizational strategies is mediated by working memory capacity
}

Citation for published version (APA):

Schleepen, T. M. J., \& Jonkman, L. M. (2012). Children's use of semantic organizational strategies is mediated by working memory capacity. Cognitive Development, 27(3), 255-269.

https://doi.org/10.1016/j.cogdev.2012.03.003

Document status and date:

Published: 01/01/2012

DOI:

10.1016/j.cogdev.2012.03.003

Document Version:

Publisher's PDF, also known as Version of record

Document license:

Taverne

Please check the document version of this publication:

- A submitted manuscript is the version of the article upon submission and before peer-review. There can be important differences between the submitted version and the official published version of record.

People interested in the research are advised to contact the author for the final version of the publication, or visit the DOI to the publisher's website.

- The final author version and the galley proof are versions of the publication after peer review.

- The final published version features the final layout of the paper including the volume, issue and page numbers.

Link to publication

\footnotetext{
General rights rights.

- You may freely distribute the URL identifying the publication in the public portal. please follow below link for the End User Agreement:

www.umlib.nl/taverne-license

Take down policy

If you believe that this document breaches copyright please contact us at:

repository@maastrichtuniversity.nl

providing details and we will investigate your claim.
}

Copyright and moral rights for the publications made accessible in the public portal are retained by the authors and/or other copyright owners and it is a condition of accessing publications that users recognise and abide by the legal requirements associated with these

- Users may download and print one copy of any publication from the public portal for the purpose of private study or research.

- You may not further distribute the material or use it for any profit-making activity or commercial gain

If the publication is distributed under the terms of Article $25 \mathrm{fa}$ of the Dutch Copyright Act, indicated by the "Taverne" license above, 


\title{
Children's use of semantic organizational strategies is mediated by working memory capacity
}

\author{
Tamara M.J. Schleepen*, Lisa M. Jonkman \\ Department of Cognitive Neuroscience, Section of Developmental Cognitive Neuroscience, Faculty of Psychology and Neuroscience, \\ Maastricht University, The Netherlands
}

\section{A R T I C L E I N F O}

\section{Keywords:}

Strategy use

Memory organizational strategies

Memory development

Working memory capacity

Short-term memory

\begin{abstract}
A B S T R A C T
In adults, the ability to apply semantic grouping strategies has been found to depend on working memory. To investigate this relation in children, two sort-recall tasks (one without and one with a grouping instruction) were administered to 6-12-year-olds. The role of working memory was examined by means of mediation analyses and by assessing whether children who successfully used the semantic grouping strategy had higher working memory capacity than did children who did not show such strategy use. Only children aged 8-12 were able to successfully use semantic grouping strategies (and 8-9-year-olds only after instruction), while strategy use was absent in 6-7-year-olds. Both types of analysis involving working memory suggested that, also in children, working memory (and not short-term memory) mediates the development of successful use of the semantic grouping strategy during both encoding and retrieval.
\end{abstract}

(c) 2012 Elsevier Inc. All rights reserved.

Storage of information in long-term memory (LTM) can be improved by the use of strategies that organize information in working memory (WM) prior to encoding. Storage of information in meaningful groups facilitates later retrieval (Dehn, 2008; Shiffrin \& Atkinson, 1969; Tulving, 1962). Such groupings may be based on perceptual similarities between items, such as a similar color or shape, or on semantic relatedness, such as belonging to the semantic category "animals" (Lange, Guttentag, \& Nida, 1990; Melkman, Tversky, \& Baratz, 1981). Effective employment of grouping strategies aids children both in daily activities and in academic contexts. For example, children's organization of basic

\footnotetext{
* Corresponding author at: Maastricht University, Faculty of Psychology and Neuroscience, Department of Cognitive Neuroscience, P.O. Box 616, 6200 MD Maastricht, The Netherlands.

E-mail address: tamara.schleepen@maastrichtuniversity.nl (T.M.J. Schleepen).
} 
math facts in LTM helps them to recall those facts later to solve larger, more complex mathematical problems (Geary \& Brown, 1991). Learning-disabled children have been reported to make less use of semantic organizational strategies in a free-recall task and show poorer recall (Bauer, 1977; Torgesen, 1977).

It is thus important to identify the factors involved in children's ability to apply intentional grouping strategies. The present study examines the role of age and conditions and, in particular, the role of working memory capacity (WMC). By about age 7, children begin to use elementary memory strategies, such as rehearsal (Ornstein, Baker-Ward, \& Naus, 1988; Ornstein \& Naus, 1978). Preschoolers spontaneously group material based on overlap in perceptual features but not on semantic relationships (Melkman et al., 1981). When items have strong, well-learned semantic associations (e.g., cow-milk), grouping strategies are reported to emerge relatively early, at around age 9. However, when items have low within-category associations (e.g., bird-dolphin), spontaneous application of the semantic grouping strategy is only reported beginning at age 13 (Bjorklund \& de Marchena, 1984; Bjorklund \& Jacobs, 1985).

Such developmental differences can be explained by differing demands on mental resources. When associations are well-learned, they are activated more or less automatically when seeing or hearing stimuli, requiring no active-grouping strategy (Schneider \& Pressley, 1997). When associations between stimuli are less well-learned, one must actively search for and encode such relationships in WM (Baddeley, 2000; Daneman \& Carpenter, 1980). Because WM has limited capacity and is subject to development, WM may affect children's ability to actively apply semantic memory-grouping strategies. Late development of this ability (Bjorklund \& de Marchena, 1984; Bjorklund \& Jacobs, 1985) parallels the recently reported prolonged maturational course of WM into adolescence (Conklin, Luciana, Hooper, \& Yarger, 2007; Diamond, Kirkham, \& Amso, 2002; Schleepen \& Jonkman, 2010). The neurobiological source of this late development has been identified as the protracted development of a network of prefrontal and parietal brain regions that undergo considerable structural and functional changes throughout this period (Bunge \& Wright, 2007; Luna, Padmanabhan, \& O'Hearn, 2010). Furthermore, WM plays an important role in learning complex cognitive activities involving language, mathematics, and reasoning (Andersson \& Lyxell, 2007; Barrouillet \& Lepine, 2005; Noel, 2009; Pickering, 2006), perhaps involving strategy use.

A link between WM and the productive application of grouping strategies has been shown in adults. Individuals with high WM span are more likely than those with a low span to employ a semantic clustering strategy when retrieving information from LTM in a verbal fluency task (Rosen \& Engle, 1997), and those with more effective semantic memory strategies show superior WM (McNamara and Scott (2001).

To our knowledge, only two studies have explored relations between WM and semantic grouping in children. Developmental studies have often made use of "sort-recall" tasks (Schneider \& Pressley, 1997) that typically consists of two phases. In the first phase, children are presented with randomly ordered pictures of objects that belong to different semantic or perceptual categories. They are instructed to study these pictures for later recall and told they may move the pictures if they think it will help them remember. After a short "buffer-clearing" interval, in a second phase children are asked to verbally report as many pictures as they can. The level of grouping on perceptual or semantic relatedness a child engages in is evaluated by computing so-called clustering scores (Roenker, Thompson, \& Brown, 1971).

Using a sort-recall task, Schneider, Kron, Hunnerkopf and Krajewski (2004) studied differences in WM among school-aged children who were consistently strategic or utilization deficient (UD) in the use of the semantic-grouping strategy. Children were assessed at two time points and classified as UD if their sorting behavior increased significantly across time without corresponding increases in recall. Consistent strategy users were those children who applied the sorting strategy at both points and demonstrated consistently high recall. UD children had lower WM scores (measured by the digit span backward task) than consistent strategy users. In a follow-up study including nine longitudinal measurement points, Kron-Sperl, Schneider, and Hasselhorn (2008) reported that individual differences in short-term memory (STM) span, but not WMC, predicted recall performance in 8-10-year-olds who spontaneously used the semantic grouping strategy. There are, however, several factors that might explain the absence of a WM-semantic memory grouping relation in this study. The to-be-grouped 
items were highly associated, so that perhaps no deliberate strategy application (requiring WM) was needed, as noted earlier.

In conclusion, there is evidence that WM affects adults' ability to deliberately apply semantic organization or grouping strategies. There is, however, a lack of information about such relations in children that may be potentially important as argued earlier. The present study investigates such relations by presenting a sort-recall task to children 6-12 years of age. The to-be-learned pictures belonged to different color or semantic categories. Only pictures with low within-category associations (e.g., dog-monkey instead of dog-cat) were used so as to examine the development of deliberate, effort-demanding, grouping-strategy application that has been shown to depend on WM in adults.

Our main goal was to study whether children who successfully implemented the semanticgrouping strategy at the encoding and retrieval phase (strategic children) had higher WMC than children who did not show such strategy use (nonstrategic children). In some studies, children were classified as "strategic" based on above-chance clustering scores during sorting only. Although sorting during study has regularly been used as a measure of conscious strategy use (Schneider \& Pressley, 1997), and earlier studies reported positive correlations between sorting scores and recall, high sorting scores alone do not assure that the sorted items were also actively encoded and retrieved in semantic groups in LTM. Sorting-related increases in recall may be caused by factors other than active encoding in semantic groups, for example by having more intensively processed the items during sorting. In the present study, we therefore classified children as strategic/nonstrategic based on their sorting behavior and also according to their use of clustering during retrieval. High semantic clustering scores during retrieval of material that is not highly associated is thought to indicate deliberate, effortful use of the grouping strategy (Bjorklund, 1987; Bjorklund, Coyle, \& Gaultney, 1992; Bjorklund \& de Marchena, 1984).

Another of the study's aims was to analyze developmental differences. At what age are normally developing children able to spontaneously use perceptual or semantic-grouping strategies and does this use lead to higher recall? Before children become able to use a memory strategy spontaneously and successfully, they may experience (at least) two types of deficiencies. During the first, mediationdeficiency phase (Reese, 1962), children do not spontaneously use a memory strategy and cannot be prompted to do so. During the second, production-deficiency phase (Flavell, 1970), children do not engage in spontaneous strategy use but are able to use it successfully after prompting. To examine the occurrence of mediation versus production deficiencies, all children performed the sort-recall task both without and with a nondirective grouping prompt. Earlier studies have shown that children aged 4-7 need elaborate training or explicit prompting about the to-be-grouped categories to be able to use and benefit from grouping strategies (Carr \& Schneider, 1991; Lange et al., 1990; Lange \& Pierce, 1992; Moeley, Olson, Halwes, \& Flavell, 1969). The present study adds to this literature by examining whether and at what age children might benefit from a single nondirective grouping prompt. It might be that from a certain age, children have sufficient skills and capacity to apply the strategy but do not spontaneously use it. These children might benefit from a single nondirective grouping prompt without requiring extensive training.

\section{Method}

\subsection{Participants}

A total of 83 participants from a primary school were divided into three age groups-6-7, 8-9 and 10-12 years; these groupings were based on the knowledge discussed earlier regarding age trends in strategy use.

Demographic characteristics (age, group size, gender, IQ and attention scores and socioeconomic status) are presented in Table 1. IQ scores were derived from a short form of the Wechsler Intelligence Scale for Children (WISC-III, Dutch version), including the vocabulary and block design subtests, that correlates .9 with the full test (Jeyakumar, Warriner, Raval, \& Ahmad, 2004; Spreen \& Strauss, 1998). Children with IQ scores below 80 were excluded. Children with diagnosed childhood disorders like ADHD, ODD, CD, autism, or learning disorders, and children taking medication, were excluded from participation. Because attention problems are highly related to WM deficits, effort was made 
Table 1

Demographic characteristics per age group.

\begin{tabular}{lccc}
\hline Variable & Age group & \\
\cline { 2 - 4 } & $6-7$ & $8-9$ & $10-12$ \\
\hline$N$ & 28 & 30 & 25 \\
Gender $^{\text {a }}$ \% female) & 50 & 57 & 64 \\
Age & $6.6(.50)$ & $8.6(.50)$ & $11.0(.82)$ \\
Attention score & $54.2(4.2)$ & $54.6(4.5)$ & $54.5(4.2)$ \\
Estimated IQ $^{\mathrm{b}}$ & $109.8(13.5)$ & $107.8(13.4)$ & $107.6(12.4)$ \\
SES $^{\mathrm{d}}$ & $5.5(2.0)$ & $6.2(1.4)$ & $5.3(1.8)$ \\
\hline
\end{tabular}

Note. Standard deviations are depicted between brackets.

a There were no significant differences in gender between age groups $\left(\chi^{2}(2)=1.05, p>.1\right)$.

b None of the participants scored above the clinical threshold on the attention subscale of the CBCL. CBCL attention scores did not significantly differ between age groups $(F(2,82)=.072, p>.1)$.

c IQ did not significantly differ between age groups $(F(2,82)=.25, p>.1)$.

d Socioeconomic status (SES) was determined by Hollingshead (1975) occupational scale for the parent holding the higher status job ( 1 or 2 = unskilled or unemployed positions, 3 or 4 = skilled or semiskilled labourers, 5 or $6=$ managerial professions, 8 or $9=$ major professions). Parental occupation data was not available for one child in each age group. SES was not significantly different between age groups $(F(2,79)=1.8, p>.1)$.

to exclude undiagnosed attention deficits by allowing parents to fill out the Child Behavior Checklist (CBCL; Achenbach, 1991). Children scoring above the clinical threshold on the attention subscale were excluded. A total of 0 children were excluded from participation based on one or more of these criteria.

\subsection{Procedure}

Children were tested in a quiet room at their school in a single experimental session lasting approximately $2 \mathrm{~h}$. Tasks were administered in a constant order. In addition to the tasks reported on here, children performed a computerized WM task that was administered in two separate parts. Each session began with the first 4 blocks of a computer-presented memory task, followed by the first sort-recall task. No grouping instructions were provided, and the child's performance was intended to reflect spontaneous use of grouping strategies. After children completed the remaining 3 blocks of the memory task, a second sort-recall task was presented in which children were prompted to apply a grouping strategy. At the end of the session, children completed the short-form WISC-III and digit-span forward and backward tests.

Before the start of the first sort-recall task, the cards were placed on a 3-row-by-4-column array and covered with a cloth. The layout of the cards was similar for all participants, with the restriction that no two items from the same semantic or color category lay adjacently. The experimenter uncovered the cards and children were instructed to label the pictures to verify that they were familiar with each object. If a child did not know an object, the experimenter provided the correct name but took care not to provide any reference to the corresponding semantic category. Because all of the pictures were chosen based on familiarity rates for 5-6-year-olds (Cycowicz, Friedman, Rothstein, \& Snodgrass, 1997), it was rare that a child did not know the correct name of a picture. Children were then instructed to remember as many pictures as possible. They were told that the pictures would be removed after a while, after which they would be asked to name as many pictures as possible. They were also told they were allowed to move the pictures in any way that might help their recall.

Following a 75-s study period, the experimenter made a photograph of the arrangement of the pictures. The experimenter then removed the pictures from the child's view and children were instructed to count up to 50 for a period of $30 \mathrm{~s}$. This served as a buffer-clearing task to control for any recency or primacy effects. Children were then asked to recall as many items as possible. Recall was voice recorded. After the first silent period of $10 \mathrm{~s}$ in which the child did not report any additional pictures, the experimenter asked whether there were any more pictures that the child could remember. After another continued silence of $15 \mathrm{~s}$, the task was ended. 
Table 2

Stimulus items per category in the two sort-recall tasks.

\begin{tabular}{lll}
\hline Semantic category & Standard task & Instruction task \\
\hline Fruit & Peer (Pear) & Ananas (Pineaple) \\
& Aardbei (Strawberry) & Kers (Cherry) \\
& Citroen (Lemon) & Meloen (Melon) \\
\multirow{3}{*}{ Animals } & Druiven (Grapes) & Appel (Apple) \\
& Dolfijn (Dolphin) & Kat (Cat) \\
& Hond (Dog) & Olifant (Elephant) \\
& Vogel (Bird) & Koe (Cow) \\
Clothes & Aap (Monkey) & Zwaan (Swan) \\
& Jas (Coat) & Hoed (Hat) \\
& Rok (Skirt) & Broek (Trousers) \\
& Das (Tie) & Want (Glove) \\
& Sok (Sock) & Jurk (Dress) \\
\hline
\end{tabular}

The second sort-recall task was identical with the exception that, before the start of this task, children were told that it might be easier to remember the pictures if they placed the pictures in groups that belong together. This instruction is considered nondirective because no hints were given as to the perceptual or semantic categories into which pictures could be grouped. At the end of this task, children were checked for color-blindness by asking them to label the four colors that were used.

\subsection{Tasks}

\subsubsection{Sort-recall task}

The material for the sort-recall task comprised two stimulus sets of 12 black-and-white line drawings of objects printed on $6 \mathrm{~cm} \times 5 \mathrm{~cm}$ cards. The borders of the cards $(0.2 \mathrm{~cm})$ were printed in one of four colors (green, blue, brown or gray). The objects in each set could be grouped according to semantic category (four objects from three distinct categories; see Table 2) and/or by color. For each stimulus set, different pictures of objects (from the same semantic categories) were used, and the two stimulus sets were counterbalanced across the two sort-recall tasks and across age groups.

The choice of line drawings in the two stimulus sets was based on several considerations. First, semantic categories of stimuli were selected that were common to children within this age range. This selection was undertaken based on familiarity rates for 5- and 6-year-olds (Cycowicz et al., 1997). This procedure yielded highly and equally familiar objects from the three semantic categories. Second, only object stimuli were included that consisted of 3-7-letter words. This restriction ensured that differences in recall performance could not be attributed to the fact that short-item words are better recalled than long-item words (Neath, Bireta, \& Surprenant, 2003). Finally, the least-associated items within each category were selected to constitute the two stimulus sets. This selection was accomplished by means of a pilot study with adults in which all possible combinations of pairings of two pictures from the same semantic category were presented to a group of 13 adults. These adults rated the level of association between items on a 10 -point scale ( $1=$ low association and $10=$ high association). Mean association scores for all pairs per category fell between 2.3 and 2.7 (SD between 1.5 and 1.9). Adults were instructed that the extent of association could be based on different features and that the ratings should be based on their first impressions. The rationale for including adults in this pilot study was that if adults, with their elaborate knowledge bases, were to rate item pairs as having a low semantic association, one can assume this would be the case for children.

\subsubsection{Short-term memory and working memory tasks}

The digit-span forward and digit-span backward tests, adapted from the Wechsler Intelligence Scale for Children (WISC-III, Dutch version), were used as measures of STM and WMC (Gathercole, Pickering, Ambridge, \& Wearing, 2004; St Clair-Thompson, 2010). 


\section{Table 3}

Mean semantic sorting and retrieval clustering scores and percentages of children showing meaningful strategy use (ARC scores $\geq .5$ ) by age and instruction condition.

\begin{tabular}{|c|c|c|c|c|c|c|}
\hline & \multicolumn{6}{|l|}{ Age group } \\
\hline & 6-7 score & Percent & 8-9 score & Percent & 10-12 score & Percent \\
\hline Short-term memory & $6.5(1.4)$ & & $6.8(1.4)$ & & $7.9(1.3)$ & \\
\hline Working memory & $3.4(1.0)$ & & $4.7(1.4)$ & & $5.3(2.0)$ & \\
\hline \multicolumn{7}{|l|}{ Standard task } \\
\hline Sorting & $-.13(.55)$ & $18 \%$ & $-.08(.58)$ & $23 \%$ & $.41(.69)$ & $60 \%$ \\
\hline Retrieval & $.01(.53)$ & $18 \%$ & $-.01(.51)$ & $17 \%$ & $.52(.61)$ & $68 \%$ \\
\hline Recall & $7.6(2.3)$ & & $8.2(2.0)$ & & $10.5(1.8)$ & \\
\hline \multicolumn{7}{|l|}{ Instructed task } \\
\hline Sorting & $.27(.61)$ & $46 \%$ & $.59(.60)$ & $70 \%$ & $.86(.36)$ & $92 \%$ \\
\hline Retrieval & $.21(.56)$ & $39 \%$ & $.51(.51)$ & $57 \%$ & $.76(.32)$ & $80 \%$ \\
\hline Recall & $6.6(2.4)$ & & $8.8(2.3)$ & & $10.3(1.5)$ & \\
\hline
\end{tabular}

Note. Short-term memory is assessed by the WISC forward digit span task and Working memory by the WISC backward digit span task.

\subsection{Scoring strategy use}

The procedures for scoring strategy use were similar to those used in most prior research. Adjusted ratio of clustering (ARC) scores (Roenker et al., 1971) were used to assess the amount of color or semantic sorting during study and clustering at retrieval. The ARC score does not vary systematically with the number of recalled items (Murphy, 1979), making it an appropriate measure of strategy use when different levels of recall are expected for different groups, as in the present case. In the present study two types of clustering (ARC) scores are calculated. First, the sorting ARC score that reflects strategy use during the study phase of the task and reflects the extent to which pictures from the same color or semantic category are sorted in successive order above a certain chance level. Second, we calculated an ARC retrieval clustering score by counting the words belonging to the same color or semantic category that were recalled in successive order above a certain chance level during the retrieval phase of the task. ARC scores vary between -1 and 1 , with a score of 1 reflecting perfect sorting or retrieval clustering, a score of zero indicating sorting or retrieval clustering at chance level, and a score below zero representing sorting or retrieval clustering below chance level. In calculating the ARC sorting score, a repetition was counted if two pictures of the same semantic category or color were laid above, below or next to one another. Intrusions and perseverations were not included in calculating the ARC retrieval scores. Following Coyle and Bjorklund (1997), sorting during study or clustering at retrieval was considered to be meaningful (i.e., children were considered strategic) when clustering scores were equal to or greater than .5. An ARC score of .5 reflects a value of slightly more than one standard deviation greater than sorting or clustering expected by chance.

\section{Results}

Due to non-normal distributions, all data were log-transformed prior to statistical analysis. Because no meaningful patterns appeared for color sorting or retrieval (ARC scores $<5$ ), only analyses for semantic sorting and retrieval are reported.

\subsection{Developmental differences in the sort-recall task}

Table 3 presents mean ARC scores for semantic sorting during study and clustering during retrieval, mean recall, and percentage who sorted or clustered above chance levels (ARC scores $\geq .5$ ), as a function of age and instruction condition. Mixed design repeated measures ANOVA's were performed with age (6-7, 8-9, and 10-12) as between-subjects factor and instruction (no instruction, grouping instruction) as within-subjects factor to study development of strategy use and effects of instruction. 


\subsubsection{Sorting during study}

Analysis of semantic sorting-scores during study yielded main effects of instruction (no instruction vs. grouping instruction) and age group (6-7, 8-9, and 10-12), $F(2,80)=45.8, p<.00001, \eta_{\mathrm{p}}^{2}=.36$, and $F(2,80)=9.5, p=.001, \eta_{\mathrm{p}}^{2}=.19$, respectively. Semantic sorting was higher in the instructed task than in the non-instructed standard task $(p<.00001)$. Post hoc analyses showed that semantic sorting scores were higher among 10-12-year olds than among either 8-9-year-olds or 6-7-year-olds $(p<.05$ and $p<.001$, respectively), who did not significantly differ from each other $(p=.45)$. Mean group ARC scores (Table 3 ) indicate that, before instruction, semantic sorting approached meaningful levels only among 10-12-year-olds. After instruction, both 8-9-year-olds and 10-12-year-olds had meaningful ARC sorting scores, whereas 6-7-year-olds did not sort meaningfully before or after instruction.

\subsubsection{Clustering at retrieval}

For clustering at retrieval, main effects of instruction and age were found, $F(2,80)=15.5, p<.001$, $\eta_{\mathrm{p}}^{2}=.16$, and $F(2,80)=12.9, p<.0001, \eta_{\mathrm{p}}^{2}=.25$, respectively. Semantic clustering at retrieval was higher in the instructed task than in the non-instructed task $(p<.001)$. Scores were higher among 10 -12-year-olds than 8-9-year-olds or 6-7-year-olds ( $p<.01$ and $p<.00001$, respectively), and the latter two groups did not differ significantly from one another $(p=.51)$. Before instruction, semantic ARC retrieval scores were meaningful (ARC $\geq .5$ ) only in 10-12-year-olds (Table 3 ). After instruction, both 8-9-year-olds and 10-12-year-olds had meaningful ARC retrieval scores, while scores were below chance levels among 6-7-year-olds both before and after instruction.

\subsubsection{Recall}

A significant age $\times$ instruction interaction was found for recall scores, $F(2,80)=3.6, p<.05, \eta_{\mathrm{p}}^{2}=.08$. A main age effect appeared for the non-instructed task, $F(2,80)=11.8, p<.0001, \eta_{\mathrm{p}}^{2}=.23$. The $10-12$ year-olds showed higher recall than either $6-7$ - or 8 -9-year-olds ( $p<.0001$ and $p<.01$, respectively). Recall performance did not significantly differ between $6-7$ - and 8-9-year-olds $(p=.59)$. For the instructed task, a main effect of age was found, $F(2,80)=17.6, p<.00001, \eta_{\mathrm{p}}^{2}=.31$. The 8-9-yearolds had recall scores similar to $10-12$-year-olds $(p=.13)$. Both groups showed higher recall than $6-7$-year-olds ( $p<.001$ and $p<.00001$, respectively).

\subsection{Relations among age, strategy use and recall}

Correlations are presented in Table 4. Significant positive correlations between ARC sorting and recall scores and between ARC retrieval and recall scores were found in 10-12-year-olds in both standard and instructed task (except that the correlation between ARC sorting and recall was not significant in the instructed task). In 8-9-year-olds, these correlations only reached significance in the instructed task, while 6-7-year-olds showed no significant correlations in either task. ARC sorting scores were positively correlated with ARC retrieval scores in all age groups in both standard and instructed tasks, except that among 6-7-year-olds, this correlation was non-significant in the standard task.

In sum, neither of the instruction conditions led to above-chance semantic sorting or retrieval clustering scores among the youngest children, indicating that they were mediation deficient. The 8-9-year-olds did not spontaneously sort or cluster according to semantic category but had abovechance sorting and retrieval clustering scores after having received a nondirective grouping prompt. This prompt was also related to higher recall, showing they were production deficient. The 10-12year-olds had above-chance sorting and retrieval-clustering scores in both instruction conditions that were consistently related to higher recall, demonstrating that they were consistently strategic.

\subsection{The role of STM/WM in successfully using semantic grouping strategies}

To investigate the role of STM and/or WMC in the successful use of the semantic grouping strategy, we performed two types of analyses. First, we performed mediation analyses to examine whether STM/WMC is a significant mediating factor in the relationship between semantic strategy use and 
Table 4

Pearson's correlations between sorting during study, clustering at retrieval, recall and STM and WM by task and age group.

\begin{tabular}{lccc}
\hline & Age group & & \\
\cline { 2 - 4 } & $6-7$ & $8-9$ & $10-12$ \\
\hline STM-WM & -.02 & .15 & .03 \\
Standard task & & & $.45^{*}$ \\
$\quad$ Sorting-clustering & .30 & $.46^{* *}$ & $.59^{* *}$ \\
Sorting-recall & .16 & .24 & $.61^{* *}$ \\
Clustering-recall & -.15 & .10 & .22 \\
STM-sorting & -.01 & .25 & .01 \\
STM-clustering & -.09 & $.46^{*}$ & .32 \\
STM-recall & -.04 & .01 & .09 \\
WM-sorting & .15 & .19 & .21 \\
WM-clustering & -.05 & .08 & $.38^{* * *}$ \\
WM-recall & .08 & .23 & $.54^{* *}$ \\
Instruction task & & & .07 \\
Sorting-clustering & $.49^{* *}$ & $.52^{* *}$ & $.45^{*}$ \\
Sorting-recall & .25 & $.42^{*}$ & .24 \\
Clustering-recall & .13 & $.40^{*}$ & .28 \\
STM-sorting & -.10 & .23 & .24 \\
STM-clustering & .02 & -.04 & -.02 \\
STM-recall & -.09 & -.07 & .20 \\
WM-sorting & $.40^{*}$ & -.24 & $.42^{*}$ \\
WM-clustering & .20 & -.00 & .07 \\
WM-recall & .24 &
\end{tabular}

Note. STM: short-term memory; WM: working memory.

${ }^{*} p<.05$.

${ }^{* *} p<.01$.

*** $p=.064$ (trend-significant).

recall. Second, we performed a between-group analysis to test whether children who successfully used the semantic grouping strategy had higher STM and/or WMC than children who did not use this strategy.

\subsubsection{Mediation analyses}

We followed recommendations from Baron and Kenny (1986), who stated that a full mediation analysis involves studying the following three relations: (1) between the independent variable and the mediator, (2) between the independent variable and the dependent variable, and (3) between the mediator and the dependent variable after controlling for the independent variable. If these are significant, the Sobel test (Sobel, 1982) can be performed to determine whether the effect of the independent variable on the dependent variable is significantly less when the effect of the mediator is taken into account. The mediation analysis was performed over the full sample $(n=83)$, based on recommendations by Mackinnon, Warsi and Dwyer (1995) that a minimal sample size of 50 is required to obtain sufficient power to detect significant mediation effects. To include both spontaneously strategic and production deficient children, the mediation analysis was performed on data from the instructed condition.

For STM, correlations for both sorting and retrieval showed that not all three criteria were met to perform the Sobel test (for sorting, the first correlation was not significant, while for retrieval clustering, the third correlation was not significant). Thus, STM did not mediate the relationship between sorting and recall or between clustering at retrieval and recall. Concerning WMC, for the sorting strategy a borderline significant correlation was found between ARC scores and WMC $(r=.20, p=.074)$ and a significant correlation between ARC scores and recall $(r=.42, p<.0001)$ and between WMC and recall after controlling for sorting $(r=.38, p<.001)$. Despite the borderline-significant first correlation, the Sobel test was performed, revealing that WMC did not mediate the relation between sorting and recall, $z=1.58, p>$.1. For the clustering retrieval strategy, significant correlations were found between (1) ARC retrieval scores and WMC $(r=.31, p<.01)$, (2) ARC retrieval scores and recall $(r=.51, p<.00001)$, and 


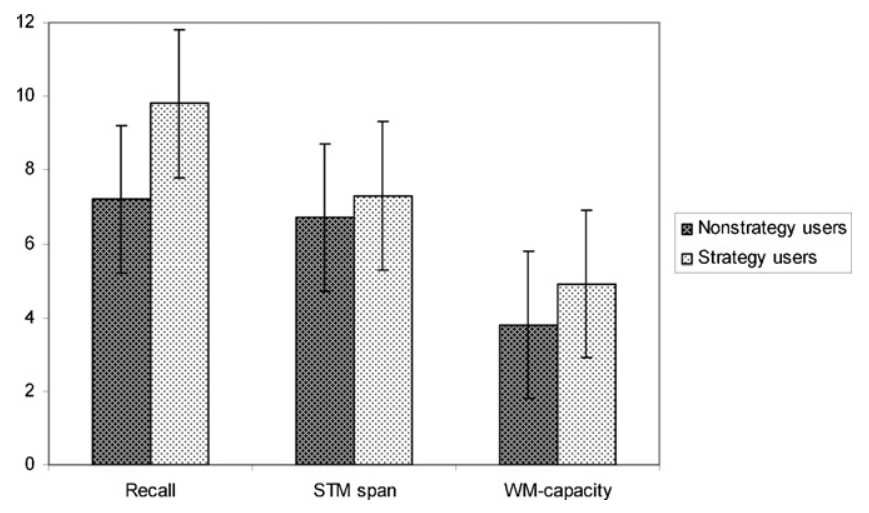

Fig. 1. Mean recall, STM and WM scores among strategy users and nonstrategy users.

(3) WMC and recall after controlling for clustering at retrieval $(r=.33, p<.01)$. As all three criteria for mediation were met, the Sobel test was performed and showed a significant mediation effect, $z=2.05$, $p<.05$, indicating that WMC was a significant mediator in the relation between clustering at retrieval and recall.

\subsubsection{Differences in STM/WMC between strategy and non-strategy users}

These analyses involved several steps. First, children were divided into two groups of strategy or non-strategy users independent of age. A child was assigned to the strategic group when ARC scores were $\geq .5$ (see Table 3). Strategy groups were formed separately for sorting and clustering retrieval strategies. The assignment to strategy groups was based on sorting and retrieval clustering scores in the instructed condition so as to include production-deficient children. Second, by applying Mann-Whitney tests, we examined whether strategy users and non-strategy users differed in their recall performance and STM/WMC. Third, the relationship between recall and STM or WMC was examined by computing correlations between recall and digit-span forward (STM) and backward (WM) scores separately for strategy and non-strategy users. For all analyses, two-tailed significance levels of $p<.05$ were adopted.

For the sorting strategy, there were 57 strategic and 26 non-strategic children. For the clustering retrieval strategy, there were 48 strategic and 35 non-strategic children. Mann-Whitney tests showed significantly higher recall among children who applied the sorting strategy, $U=1060, p<.01$ and children who applied the clustering retrieval strategy, $U=1276, p<.0001$, than among those who did not. Furthermore, whereas forward digit-span scores were borderline higher among strategic sorters compared to nonstrategic sorters, $U=932, p=.056$, forward digit-span scores did not differ between children who did or did not apply the clustering retrieval strategy, $U=981, p>.1$ (see Fig. 1 ). In contrast, backward digit-span scores did not significantly differ between children who did or did not use the sorting strategy, $U=855, p>.1$, while children who applied the clustering retrieval strategy had significantly higher backward digit-span scores than children who did not use this strategy, $U=1088$, $p<.05$ (Fig. 1).

Again, there were no significant correlations between STM and recall in the strategic or nonstrategic groups ( $p s>.1$ ). As expected, WMC did correlate significantly with recall among children who successfully applied the clustering retrieval strategy $(r=.40, p<.01)$ but not in non-strategic clusterers $(p>.1)$. The WM-recall relationship was also significant among the group of strategic sorters $(r=.45, p<.001)$ while not among non-sorters $(p>.1)$. This finding is likely due to the large overlap (42 participants) between strategic sorting and clustering retrieval groups. A hierarchical regression analysis in the group of strategic sorters, with WMC entered in the first block and ARC sorting and ARC retrieval scores in the second block, showed that WMC explained $20 \%$ $(p<.001)$ and retrieval clustering an additional 21.6\% $(p<.0001)$ of the variance in the recall scores of the strategic sorters, whereas sorting did not explain any additional variance. Fig. 2 shows the 

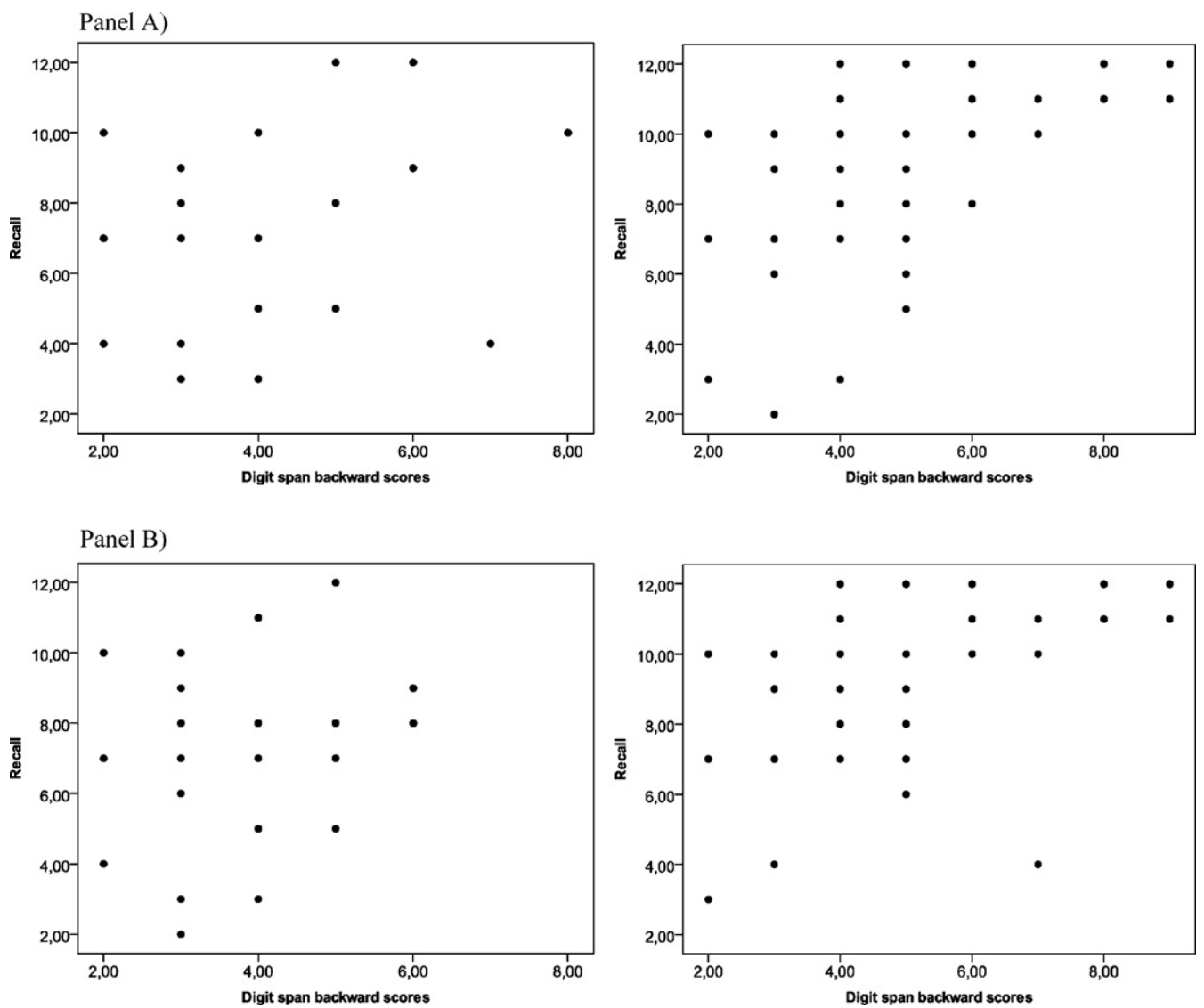

Fig. 2. Correlation between recall and WMC in nonstrategy users (Panel A, left) and strategy users (Panel A, right) for sorting. Some points represent two or more participants. Cook's $D$ was calculated to trace potential outliers that may have influenced the correlations. This revealed three cases with large Cook's $D$ values in strategic children. Excluding these cases did not substantially lower the correlation between WMC and recall $(r=.42, p<.01)$. The correlation between recall and WMC for retrieval clustering appears in Panel B (left for nonstrategy users and right for strategy users). Three cases with large Cook's $D$ values appeared in strategic children. Excluding these cases did not substantially lower the correlation between WMC and recall $(r=.46, p<.01)$.

scatter-plots that depict the correlation between WMC and recall in all strategic and nonstrategic groups.

Finally, to also investigate the role of STM and WMC in the prediction of recall performance above strategy use in the entire sample, we performed a regression analysis entering semantic sorting and clustering retrieval scores in the first step and STM and WM scores in the second step. This analysis demonstrates that WMC (but not STM) additionally explains $9.2 \%(p<.01)$ of the variance in recall scores above semantic sorting and clustering retrieval scores.

\section{Discussion}

The primary aim of the present study was to investigate whether working memory is an important determinant of the efficient use of semantic grouping strategies in children. A second aim was to investigate the mean age at which children begin to spontaneously use color or semantic grouping strategies that improve memory, along with the age at which a single nondirective grouping instruction leads to the successful application of the strategy in non-spontaneous users. This was investigated 
by having 6-12-year-old children perform a sort-recall task with pictures that belonged to different color or semantic categories. The tasks were performed twice, once without and once with a nondirective prompt in which children were told that grouping pictures that belong together might improve recall (the categories were not mentioned).

Results showed that only 10-12-year-olds were able to spontaneously and successfully use the semantic grouping strategy. Semantic grouping strategy use was absent in 6-7-year-olds, while children aged 8-9 only (successfully) used this strategy after prompting. The role of STM and/or WMC (as inferred from forward and backward digit-span tests, respectively) was investigated by performing mediation analyses and testing whether strategy users had higher recall and higher STM/WM than non-strategy users. Both types of analysis suggested that WMC (and not STM) mediates the development of successful use of the semantic grouping strategy.

\subsection{Developmental differences in the use of semantic grouping strategies}

Color-grouping scores during sorting and retrieval were below chance levels in all age groups and will not be further discussed. Developmental differences in the deliberate application of the semantic organizational strategy and the role of WM were investigated by only including items that had low associations within their category (Bjorklund \& de Marchena, 1984; Bjorklund \& Jacobs, 1985). During the study phase, the categorization of less-associated items into semantic categories demanded that children consciously and deliberately search for categories and to subsequently actively encode the items in these categories. These categories could then be used as retrieval cues to facilitate later recall. By comparing sorting, clustering retrieval and recall scores across age groups in the sort-recall task without instruction, information was obtained regarding the age at which children showed spontaneous strategy use. Below-chance ARC sorting and clustering retrieval scores were found in 6-7-year-olds and 8-9-year-olds, signifying that they did not spontaneously sort or retrieve the pictures in semantic categories. In contrast, among 10-12-year-olds, sorting and retrieval-clustering scores were above-chance level, and sorting, clustering and recall scores were significantly higher than among 6-9-year-olds. Moreover, sorting, clustering retrieval and recall scores significantly correlated with each other only among 10-12-year-olds, demonstrating that strategy use was successful. After receiving a non-directive grouping prompt, the 6-7-year-olds still showed no meaningful sorting during study or retrieval, and their recall performance was significantly lower than that of older children. However, among (spontaneously nonstrategic) 8-9-year-olds, the non-directive grouping prompt caused them to become as strategic as the (spontaneously strategic) 10-12-year-olds. Additionally, positive correlations between sorting during study, clustering at retrieval and recall appeared in both 8-9- and 10-12-year-olds.

Integrating the results from both instruction conditions, it can be concluded that 6-7-year-olds were mediation deficient: they did not spontaneously use memory strategies and could not be prompted to do so (Bjorklund, Miller, Coyle, \& Slawinski, 1997). In contrast, 8-9-year-olds were production deficient: they only used the strategy after prompting but with recall benefits (for review, see Schneider \& Pressley, 1997). Finally, 10-12-year-olds were consistently strategic, also without prompting.

The finding of the absence of spontaneous use of the semantic grouping strategy before age 9 is consistent with earlier findings (Bjorklund \& de Marchena, 1984; Bjorklund \& Jacobs, 1985). The start of spontaneous semantic grouping from age 10 is in line with a study by Moeley, Olson, Halwes, and Flavell (1969) but in contrast with findings by Bjorklund and colleagues (Bjorklund \& de Marchena, 1984; Bjorklund \& Jacobs, 1985), who reported effective use of semantic organization strategies with loosely associated materials only beginning at age 13 . Schneider, Borkowski, Kurtz and Kerwin (1986) reported that 9-year-old German children, but not 9-year-old American children, were able to spontaneously use the semantic organization strategy. These different findings might indicate that European children show earlier emergence of the semantic grouping strategy, possibly due to differences in teaching practices or metacognitive awareness (Kurtz, Schneider, Carr, Borkowski, \& Rellinger, 1990).

The present results add to the memory-strategy training literature by demonstrating that, whereas 8-9-year-olds do not use semantic organization strategies spontaneously, they do not need extensive 
training in strategy use to do so effectively; a single prompt to group pictures that belong together appears to be sufficient at this age. Children younger than 8 years, however, may need more extensive training to achieve semantic grouping skills. This finding is consistent with the results of earlier studies that have demonstrated that with elaborate training, including demonstration and practice in using semantic features to encode and retrieve information, as well as receiving feedback, 4-7-year-olds were able to apply semantic organization strategies and demonstrate enhanced recall (Carr \& Schneider, 1991; Lange et al., 1990; Lange \& Pierce, 1992; Stevens, 2002). The reason that a single non-directive grouping prompt only triggered strategy use from age 8 onward might be that around this age, important development in awareness about representations and their logical relatedness is taking place (Demetriou \& Kazi, 2006; Demetriou, Spanoudis, \& Mouyi, 2011).

\subsection{Roles of short-term and working memory in children's effective semantic strategy use}

Two types of analyses were performed to answer the main research question of the degree to which STM and/or WMC contribute to the ability to successfully apply semantic grouping strategies. Both indicated that, while the successful application of the sorting strategy placed no significant demands on WMC (or STM) in 6-12-year-olds, successful application of the clustering-retrieval strategy was dependent on WMC and not on STM. This conclusion was based on the following findings: (1) STM neither mediated the relation between sorting and recall nor between clustering at retrieval and recall, (2) WM only mediated the relation between clustering at retrieval and recall, and not between sorting during study and recall, (3) WMC (not STM) was significantly higher in children who successfully applied the semantic grouping strategy during retrieval than in children who did not do so, while there were no significant WMC or STM differences between sorters and non-sorters and (4) WMC correlated with recall in both the strategic sorting and clustering retrieval groups (but not in the two non-strategic groups). However, there was a large overlap among strategic sorters and clusterers (73\% of the strategic sorters also used the semantic clustering strategy during retrieval), and a regression analysis indicated that only clustering-retrieval scores (not sorting scores) explained additional variance in recall above WM-span scores in sorters. The finding that the application of the sorting strategy consumes little of children's WM resources is in line with a study by Kron-Sperl et al. (2008), who reported that STM (not WMC) significantly predicted recall in children who consistently applied the sorting strategy. In our study, STM differences between sorters and non-sorters were only borderline significant. The low dependency of sorting on WMC might mean that with loosely associated materials, sorting does not automatically lead to active encoding (and subsequent retrieval) of items in semantic clusters. Thus far, WM has been found to play an important role in semantic memory strategy use in adults (Gaultney, Kipp, \& Kirk, 2005; McNamara \& Scott, 2001; Rosen \& Engle, 1997) and in cumulative rehearsal strategies in children (Lehmann \& Hasselhorn, 2007). The present findings add to this literature by demonstrating the importance of WMC in the efficient use of semantic organization strategies during encoding and retrieval among children.

The present data do not answer the question of how WM mediates recall in children who make efficient use of semantic organization strategies. One can, however, speculate in this regard on the basis of prior studies in adults. During the encoding of categorized word lists, the central executive component of WM (Baddeley \& Hitch, 1974) is thought to play a role in mediating organizational strategies (Gershberg \& Shimamura, 1995) and in maintaining constant conscious awareness of the to-be-encoded targets (Moscovitch, 1992, 1994). During the retrieval of categorized lists, the central executive is only needed during controlled search for earlier stored category names, which would then automatically trigger the category items (Cinan, 2003; Rosen \& Engle, 1997). Dual-task studies in adults that manipulated available executive resources showed that when the number of to-be-retrieved category names is low, minimal demands are placed on the central executive during retrieval (Cinan, 2003; Johnston, Rollie, \& Griffith, 1972). During encoding, however, the effects of performing a secondary task that consumed central executive resources was much stronger and always compromised recall, independent of the number of categories in the to-be-learned list (Cinan, 2003). In light of these results, we hypothesize that WM influences children's recall by mediating the influences on strategy use and attention regulation during the encoding phase of the task. Future work, manipulating categories and the availability of resources could further examine this hypothesis. 
Whereas the present results show that children need sufficient WMC to be able to apply semantic grouping strategies with success (i.e., with recall benefits), other developmental studies suggest that improvements in WM performance or cognitive control are in themselves caused by developmental changes in cognitive flexibility or increased levels of consciousness. For instance, Camos and Barrouillet (2011) concluded that improvements in WM performance between 7 and 9 years of age were due to increases in cognitive (attentional) flexibility enabling older children to regularly switch attention between maintenance and processing, preventing the decay of information with time. The same cognitive flexibility that is needed in WM tasks might be necessary to switch between the processing of the items in categories and the maintenance (rehearsal) of already-stored items in the sort-recall task. Zelazo (2004) introduced his levels of consciousness (LOC) model that describes age-related changes in the degree of self-reflection that children can display, which is thought to have consequences for the potential for recall and the complexity of children's explicit knowledge structures. When children do not enter a certain (higher) level of consciousness on their own, they might be triggered to do so if provided with labels or prompts. Applying this model to our findings, the grouping prompt might have triggered 8-9-year-olds to reflect on their representations at a higher consciousness level, making them aware of the semantic associations, which they subsequently encoded. The system might have been insufficiently mature among 6-7-year-olds to achieve a higher level of consciousness based on our non-specific prompt.

Effort-demanding memory strategies such as semantic organization are fundamental for academic learning and everyday functioning. Those undertaking interventions to enhance children's use of such strategies might determine whether children have weaknesses in WM. If so, training can be aimed at increasing WMC (Dahlin, Backman, Neely, \& Nyberg, 2009; Klingberg, 2010; Morrison \& Chein, 2011), thereby promoting acquisition of efficient organizational memory skills. Also, remedial teachers should screen for WM impairments at an early age so that training can be introduced if indicated. Such efforts are important because children who are able to use more efficient strategies may have an advantage in the learning and application of cognitive skills (Bauer, 1977).

\section{References}

Achenbach, T. M. (1991). Manual for the child behavior checklist/4-18. Burlington: University of Vermont, Department of Psychiatry.

Andersson, U., \& Lyxell, B. (2007). Working memory deficit in children with mathematical difficulties: A general or specific deficit? Journal of Experimental Child Psychology, 96, 197-228.

Baddeley. (2000). The episodic buffer: A new component of working memory? Trends in Cognitive Sciences, 4, 417-423.

Baddeley, \& Hitch, G. J. (1974). Working memory. In G. A. Bower (Ed.), Recent advances in learning and motivation (pp. 47-89). New York: Academic Press.

Baron, R. M., \& Kenny, D. A. (1986). The moderator-mediator variable distinction in social psychological research: Conceptual, strategic, and statistical considerations. Journal of Personality and Social Psychology, 51(6), 1173-1182.

Barrouillet, P., \& Lepine, R. (2005). Working memory and children's use of retrieval to solve addition problems. Journal of Experimental Child Psychology, 91, 183-204.

Bauer, R. H. (1977). Memory processes in children with learning disabilities: Evidence for deficient rehearsal. Journal of Experimental Child Psychology, 24, 415-430.

Bjorklund, D. F. (1987). How age changes in knowledge base contribute to the development of children's memory: An interpretive revie. Developmental Review, 7, 93-130.

Bjorklund, D. F., Coyle, T. R., \& Gaultney, J. F. (1992). Developmental differences in the acquisition and maintenance of an organizational strategy-Evidence for the utilization deficiency hypothesis. Journal of Experimental Child Psychology, 54(3), 434-448.

Bjorklund, D. F., \& de Marchena, M. R. (1984). Developmental shifts in the basis of organization in memory: The role of associative versus categorical relatedness in children's free recall. Child Development, 55, 952-962.

Bjorklund, D. F., \& Jacobs, J. W. (1985). Associative and categorical processes in children's memory: The role of automaticity in the development of organization in free recall. Journal of Experimental Child Psychology, 39, 599-617.

Bjorklund, D. F., Miller, P. H., Coyle, T. R., \& Slawinski, J. L. (1997). Instructing children to use memory strategies: Evidence of utilization deficiencies in memory training studies. Developmental Review, 17, 411-441.

Bunge, S. A., \& Wright, S. B. (2007). Neurodevelopmental changes in working memory and cognitive control. Current Opinion in Neurobiology, 17, 243-250.

Camos, V., \& Barrouillet, P. (2011). Developmental change in working memory strategies: From passive maintenance to active refreshing. Developmental Psychology, 47, 898-904.

Carr, M., \& Schneider, W. (1991). Long-term maintenance of organizational strategies in kindergarten-children. Contemporary Educational Psychology, 16(1), 61-72.

Cinan, S. (2003). Executive processing in free recall of categorized lists. Learning and Motivation, 34, 240-261. 
Conklin, H. M., Luciana, M., Hooper, C. J., \& Yarger, R. S. (2007). Working memory performance in typically developing children and adolescents: Behavioral evidence of protracted frontal lobe development. Developmental Neuropsychology, 31, $103-128$.

Coyle, T. R., \& Bjorklund, D. F. (1997). Age differences in, and consequences of, multiple- and variable-strategy use on a multitrial sort-recall task. Developmental Psychology, 33, 372-380.

Cycowicz, Y. M., Friedman, D., Rothstein, M., \& Snodgrass, J. G. (1997). Picture naming by young children: Norms for name agreement, familiarity, and visual complexity. Journal of Experimental Child Psychology, 65, 171-237.

Dahlin, E., Backman, L., Neely, A. S., \& Nyberg, L. (2009). Training of the executive component of working memory: Subcortical areas mediate transfer effects. Restorative Neurology and Neuroscience, 27, 405-419.

Daneman, M., \& Carpenter, P. A. (1980). Individual differences in working memory and reading. Journal of Verbal Learning and Verbal Behavior, 19, 450-466.

Dehn, M. J. (2008). Working memory and academic learning: Assesment and intervention. Hoboken, NJ: Wiley \& Sons, Inc.

Demetriou, A., \& Kazi, S. (2006). Self-awareness in g (with processing efficiency and reasoning). Intelligence, 34(3), $297-317$.

Demetriou, A., Spanoudis, G., \& Mouyi, A. (2011). Educating the developing mind: Towards an overarching paradigm. Educational Psychology Review, 1-63.

Diamond, A., Kirkham, N., \& Amso, D. (2002). Conditions under which young children can hold two rules in mind and inhibit a prepotent response. Developmental Psychology, 38, 352-362.

Flavell, J. H. (1970). Developmental studies of mediated memory. In H. W. Reese, \& L. P. Lipsitt(Eds.), Advances in child development and behavior (pp. 181-211). New York: Academic.

Gathercole, S. E., Pickering, S. J., Ambridge, B., \& Wearing, H. (2004). The structure of working memory from 4 to 15 years of age. Developmental Psychology, 40, 177-190.

Gaultney, J. F., Kipp, K., \& Kirk, G. (2005). Utilization deficiency and working memory capacity in adult memory performance: Not just for children anymore. Cognitive Development, 20, 205-213.

Geary, C. G., \& Brown, S. C. (1991). Cognition addition: Strategy choice and speed of processing differences in gifted, normal, and mathematically disabled children. Developmental Psychology, 27, 398-406.

Gershberg, F. B., \& Shimamura, A. P. (1995). Impaired use of organizational strategies in free recall following frontal lobe damage. Neuropsychologia, 33, 1305-1333.

Jeyakumar, S. L. E., Warriner, E. M., Raval, V. V., \& Ahmad, S. A. (2004). Balancing the need for reliability and time efficiency: Short forms of the Wechsler Adult Intelligence Scale-III. Educational and Psychological Measurement, 64, 71-87.

Johnston, W. A., Rollie, R. W., \& Griffith, D. (1972). Information-processing analysis of verbal learning. Journal of Experimental Psychology, 96, 307-314.

Klingberg, T. (2010). Training and plasticity of working memory. Trends in Cognitive Sciences, 14(7), 317-324.

Kron-Sperl, V., Schneider, W., \& Hasselhorn, M. (2008). The development and effectiveness of memory strategies in kindergarten and elementary school: Findings from the Wurzburg and Gottingen longitudinal memory studies. Cognitive Development, 23, 79-104.

Kurtz, B. E., Schneider, W., Carr, M., Borkowski, J. G., \& Rellinger, E. (1990). Strategy instruction and attributional beliefs in WestGermany and the United-States-Do teachers foster metacognitive development. Contemporary Educational Psychology, 15, 268-283.

Lange, G., Guttentag, R. E., \& Nida, R. E. (1990). Relationships between study organization, retrieval organization, and general and strategy-specific memory knowledge in young children. Journal of Experimental Child Psychology, 49, 126-146.

Lange, G., \& Pierce, S. H. (1992). Memory-strategy learning and maintenance in preschool-children. Developmental Psychology, $28,453-462$.

Lehmann, M., \& Hasselhorn, M. (2007). Variable memory strategy use in children's adaptive intratask learning behavior: Developmental changes and working memory influences in free recall. Child Development, 78, 1068-1082.

Luna, B., Padmanabhan, A., \& O'Hearn, K. (2010). What has fMRI told us about the development of cognitive control through adolescence? Brain and Cognition, 72, 101-113.

Mackinnon, D. P., Warsi, G., \& Dwyer, J. H. (1995). A simulation study of mediated effect measures. Multivariate Behavorial Research, 30, 41.

McNamara, D. S., \& Scott, J. L. (2001). Working memory capacity and strategy use. Memory \& Cognition, 29, 10-17.

Melkman, R., Tversky, B., \& Baratz, D. (1981). Developmental trends in the use of perceptual and conceptual attributes in grouping, clustering, and retrieval. Journal of Experimental Child Psychology, 31, 470-486.

Moeley, B. E., Olson, F. A., Halwes, T. G., \& Flavell, J. H. (1969). Production deficieny in young children's clustered recall. Developmental Psychology, 1, 26-34.

Morrison, A. B., \& Chein, J. M. (2011). Does working memory training work? The promise and challenges of enhancing cognition by training working memory. Psychonomic Bulletin and Review, 18, 46-60.

Moscovitch, M. (1992). Memory and working-with-memory-A component process model based on modules and central systems. Journal of Cognitive Neuroscience, 4, 257-267.

Moscovitch, M. (1994). Cognitive resources and dual-task interference effects at retrieval in normal people: The role of the frontal lobes and medial temporal cortex. Neuropsychology, 8, 524-534.

Murphy, M. (1979). Measure of category clustering in free recall. In R. Puff (Ed.), Memory organization and structure (pp. 51-81). New York: Academic Press.

Neath, I., Bireta, T. J., \& Surprenant, A. M. (2003). The time-based word length effect and stimulus set specificity. Psychonomic Bulletin E Review, 10, 430-434.

Noel, M. P. (2009). Counting on working memory when learning to count and to add: A preschool study. Developmental Psychology, 45, 1630-1643.

Ornstein, P. A., Baker-Ward, L., \& Naus, M. J. (1988). The development of mnenomic skill. In O. A. Barbarin, \& B. H. Wasik (Eds.), Handbook of child development and early education: Research to practice (pp. 103-122). New York: Guilford.

Ornstein, P. A., \& Naus, M. J. (1978). Rehearsal processes in children's memory. In P. A. Ornstein (Ed.), Memory development in children (pp. 69-99). Hillsdale, NY: Erlbaum.

Pickering, S. J. (2006). Working memory and education. San Diego, CA: Academic Press.

Reese, H. W. (1962). Verbal mediation as a function of age level. Psychological Bulletin, 59, 502-509. 
Roenker, D. L., Thompson, C. P., \& Brown, S. C. (1971). Comparison of measures for the estimation of clustering in free recall. Psychological Bulletin, 76, 45-48.

Rosen, V. M., \& Engle, R. W. (1997). The role of working memory capacity in retrieval.Journal of Experimental Psychology-General, $126,211-227$.

Schleepen, T. M. J., \& Jonkman, L. M. (2010). The development of non-spatial working memory capacity during childhood and adolescence and the role of interference control: An N-Back task study. Developmental Neuropsychology, 35, 37-56.

Schneider, W., Borkowski, J. G., Kurtz, B. E., \& Kerwin, K. (1986). Metamemory and motivation: A comparison of strategy use and performance in German and American children. Journal of Cross-Cultural Psychology, 17, 315-336.

Schneider, W., Kron, V., Hunnerkopf, M., \& Krajewski, K. (2004). The development of young children's memory strategies: First findings from the Wurzburg Longitudinal Memory Study. Journal of Experimental Child Psychology, 88, 193-209.

Schneider, W., \& Pressley, M. (1997). Memory development between two and twenty (2nd ed.). Mahwah, NJ: Lawrence Erlbaum Associates.

Shiffrin, R., \& Atkinson, R. C. (1969). Storage and retrieval processes in long-term memory. Psychological Review, 76, $179-193$.

Sobel, M. E. (1982). Asymptotic intervals for indirect effects in structural equations models. In S. Leinhart (Ed.), Sociological methodology (pp. 290-312). San Francisco: Jossey-Bass.

Spreen, O., \& Strauss, E. (1998). A compendium of neuropsychological tests: Administration, norms and commentary. New York: Oxford University Press.

St Clair-Thompson, H. L. (2010). Backwards digit recall: A measure of short-term memory or working memory? European Journal of Cognitive Psychology, 22, 286-297.

Stevens, J. P. (2002). Applied multivariate statistics for the social sciences (4th ed.). London: Lawrence Erlbaum Associates, Publishers.

Torgesen, J. K. (1977). Memorization processes in reading-disabled children. Journal of Educational Psychology, 69, 571-578.

Tulving, E. (1962). Subjective organization in free recall of unrelated words. Psychological Review, 69, 344-354.

Zelazo, P. D. (2004). The development of conscious control in childhood. Trends in Cognitive Sciences, 8, 12-17. 